\title{
Topical nitric oxide releasing nanoparticles are effective in a murine model of dermal Trichophyton rubrum dermatophytosis
}

\author{
Breanne Mordorski, MD ${ }^{\mathrm{a}, 1}$, Caroline Barcelos Costa-Orlandi, $\mathrm{PhD}^{\mathrm{b}, \mathrm{c}, 1}$,
} Ludmila M. Baltazar, $\mathrm{PhD}^{\mathrm{b}}$, Leandro J. Carreño, $\mathrm{PhD}^{\mathrm{d}, \mathrm{e}}$, Angelo Landriscina, $\mathrm{MD}^{\mathrm{a}}$, Jamie Rosen, $\mathrm{MD}^{\mathrm{a}}$, Mahantesh Navati, $\mathrm{PhD}^{\mathrm{f}}$, Maria Jose Soares Mendes-Giannini, $\mathrm{PhD}^{\mathrm{c}}$, Joel M. Friedman, MD, $\mathrm{PhD}^{\mathrm{f}}$, Joshua D. Nosanchuk, MD ${ }^{\mathrm{b}, \mathrm{d}}$, Adam J. Friedman, MD ${ }^{\mathrm{a}, \mathrm{f}, \mathrm{g}, *}$

${ }^{a}$ Department of Medicine (Division of Dermatology), Albert Einstein College of Medicine, Bronx, NY, USA

${ }^{\mathrm{b}}$ Department of Medicine (Division of Infectious Diseases), Albert Einstein College of Medicine, Bronx, NY, USA

${ }^{\mathrm{c}}$ Departamento de Análises Clínicas, Faculdade de Ciências Farmacêuticas, Universidade Estadual Paulista (UNESP), Araraquara, SP, Brazil

${ }^{\mathrm{d}}$ Department of Microbiology \& Immunology, Albert Einstein College of Medicine, Bronx, NY, USA

${ }^{\mathrm{e}}$ Millennium Institute on Immunology and Immunotherapy, Programa de Inmunología, Instituto de Ciencias Biomédicas, Facultad de Medicina, Universidad de Chile, Región Metropolitana, Santiago, Chile

${ }_{\mathrm{f}}^{\mathrm{D}}$ Department of Physiology \& Biophysics, Albert Einstein College of Medicine, Bronx, NY, USA

${ }^{\mathrm{g}}$ Department of Dermatology, George Washington School of Medicine and Health Sciences, Washington, DC, USA

Received 28 October 2016; accepted 19 June 2017

\begin{abstract}
Systemic therapies are preferred for treating dermal dermatophytosis due to inadequate penetration of topical agents. However, systemic antifungals are associated with off-target effects and limited tissue penetration, and antimicrobial resistance is a growing concern. To address this, we investigated topical nitric oxide-releasing nanoparticles (NO-np), which have been used against superficial fungal infections and bacterial abscesses. In addition to enhanced penetration and permeation conferred by nanoparticles, nitric oxide, a broad-spectrum multimechanistic antimicrobial agent, offers decreased likelihood of resistance development. In the current study, NO-np inhibited Trichophyton rubrum in vitro, as well as in a murine model of dermal dermatophytosis. In mice, NO-np reduced fungal burden after three days, with complete clearance after seven. Furthermore, NO-np decreased tissue IL-2, 6, 10 and TNF $\alpha$, indicating earlier attenuation of the host inflammatory response and decreased tissue morbidity. Thus, topical NO-np represent an attractive alternative to systemic therapy against dermal T. rubrum infection.
\end{abstract}

(C) 2017 Elsevier Inc. All rights reserved.

Dermatophytoses afflict millions of people worldwide. Depending on infection depth and location, treatment failure may occur with both topical and systemic medications due to poor tissue penetration, side effects, lengthy treatment schedules, antifungal resistance, and failure to eliminate dormant spores. ${ }^{1}$ Although cutaneous dermatophytoses are frequently limited to the epidermis, fungi may invade into the dermis and subcutaneous tissue, especially when precipitated by trauma, immunosuppression, or use of topical corticosteroids. Deep cutaneous infection requires systemic therapy; however, conventional medications pose a range of side effects and undergo hepatic first-pass metabolism and extensive plasma protein binding, exemplifying inefficient drug delivery. Given these challenges, a topical antifungal capable of penetrating the stratum corneum and retaining potent activity in

Funding: CBCO received fellowship funding from Coordenação de Aperfeiçoamento de Pessoal de Nível Superior (process number: 99999.007910/ 2014-02) and Conselho Nacional de Desenvolvimento Científico e Tecnológico (process number: 150261/2016-0).

Disclosures: AJF and JMF are co-inventors of NO-np, a technology licensed to Nano Biomed Inc. for commercialization. JDN serves as an advisor to Nano Biomed Inc. Portions of this publication were presented in abstract form at the 74th Annual Meeting of the American Academy of Dermatology.

*Corresponding author at: Washington, DC 20037.

E-mail address: ajfriedman@mfa.gwu.edu (A.J. Friedman).

1 These authors contributed equally to the production of this work. 
deeper skin layers while evading antimicrobial resistance mechanisms is highly desired. ${ }^{2}$

Nitric oxide (NO), a gaseous immunomodulator with broadspectrum, multi-mechanistic antimicrobial activity, is an attractive agent to fill this need. Although NO is ubiquitous throughout the body, therapeutic delivery is challenging due to its short half-life and high reactivity. ${ }^{3}$ This can be overcome by a previouslycharacterized nanoparticle platform that generates and releases NO over time (NO-np). ${ }^{4-6}$ Nanoscaled particles (1-100 nm) offer enhanced cutaneous penetration and increased skin surface residence time (creating a depot effect), and their extremely large surface area increases their likelihood of interaction with fungal cells. When NO-np are exposed to moisture, NO is formed from encapsulated dinitrogen trioxide and released in a controlled, sustained manner, reaching a steady state between 184 and $196 \mathrm{ppb}$ $\mathrm{NO} / \mathrm{mg}$ within $40 \mathrm{~min}$ that lasts for several hours. ${ }^{5,7}$

A previous study with fluorescently-labeled nanoparticles indicates that NO-np penetrate the epidermal barrier and localize within hair follicles, which may serve as a reservoir for NO-np and potentiate the sustained release benefit. ${ }^{8}$ Hair follicles are also the site of dermatophytosis in Majocchi's granuloma, a specific type of deep cutaneous dermatophytosis. Thus, NO-np may offer enhanced targeting of this infection.

In other prior investigations, NO-np have exhibited activity against yeast and filamentous forms of Candida albicans, ${ }^{9}$ as well as a variety of gram-positive and -negative bacteria, ${ }^{10}$ with limited toxicity in in vitro and in vivo evaluations. ${ }^{11}$ Topical NO-np have also been used to treat murine dermal and intramuscular bacterial abscesses, deeper infections which would otherwise require systemic therapy. In abscess models, topical NO-np led to decreased bacterial burden, as well as preservation of cutaneous and muscular architecture, respectively. ${ }^{12,13}$ Given their broad activity against fungal and bacterial species, as well as previous successes in treating deeper models of infection, the current study assessed topical NO-np against Trichophyton rubrum, both in vitro as well as in a murine model of dermal dermatophytosis.

\section{Methods}

NO-np were synthesized as previously described. ${ }^{6}$ Minimum inhibitory concentrations (MICs) were determined for two T. rubrum reference strains (ATCC MYA-4438 and ATCC 28189) and a clinical isolate using methods proposed by the Clinical and Laboratory Standards Institute (CLSI), modified by the use of a resazurin colorimetric assay. ${ }^{14}$ Minimum fungicidal concentrations (MFCs) were determined as previously described. ${ }^{15}$ Additionally, antimicrobial effects of the MIC of NO-np were visualized directly by transmission electron microscopy (TEM) following $96 \mathrm{~h}$ incubation with ATCC MYA-4438.

Dermal dermatophytosis was simulated with ATCC MYA-4438 on dorsal surfaces of BALB/c mice using a previously described model. ${ }^{16}$ Seven days post-infection, mice began daily topical treatment with $100 \mu \mathrm{L}$ of either saline, coconut oil, $10 \mathrm{mg} / \mathrm{mL}$ empty nanoparticles, $10 \mathrm{mg} / \mathrm{mL} \mathrm{NO}-\mathrm{np}$, or $1 \%$ terbinafine. Nanoparticles and terbinafine were delivered in coconut oil. Following three and seven days of treatment, homogenized tissue samples were filtered with a $40 \mu \mathrm{m}$ cell strainer and plated on potato dextrose agar. Colony forming units (CFUs) were quantified after $72 \mathrm{~h}$. Following three days of treatment, cytokine analysis was performed on supernatants

\begin{tabular}{|c|c|c|c|c|c|c|c|c|}
\hline & \multicolumn{2}{|c|}{ C-np } & \multicolumn{2}{|c|}{ NO-np } & \multicolumn{2}{|c|}{ TRB } & \multicolumn{2}{|c|}{ FLZ } \\
\hline & $\underset{(\mathrm{m} \approx \mathrm{mL})}{\mathrm{NIIC}}$ & $\underset{(\mathrm{mg} \mathrm{mL})}{\mathrm{MFC}}$ & $\underset{(\mathrm{mz} \mathrm{mL})}{\mathrm{MIIC}}$ & $\underset{(\mathrm{mg} \mathrm{mL})}{\mathrm{MFC}}$ & $\begin{array}{l}\text { MIIC } \\
(\operatorname{mz} L)\end{array}$ & $\begin{array}{l}\text { MFC } \\
(\operatorname{mn} \mathbf{L})\end{array}$ & $\begin{array}{l}\text { MIIC } \\
(\mathbf{m} z \mathbf{L})\end{array}$ & $\begin{array}{l}\text { MFC } \\
(\mathrm{m}=\mathrm{L})\end{array}$ \\
\hline $\begin{array}{l}\text { Reference strain } 1 \\
\text { ATCC MIYA- }+438\end{array}$ & $>20$ & $>20$ & 10 & $>20$ & 0.03 & - & 4 & - \\
\hline $\begin{array}{l}\text { Reference strain } 2 \\
\text { ATCC } 28189\end{array}$ & $>20$ & $>20$ & 10 & $>20$ & 0.06 & - & 64 & - \\
\hline Clinical Strain & $>20$ & $>20$ & 5 & $>20$ & 0.25 & - & 32 & - \\
\hline
\end{tabular}

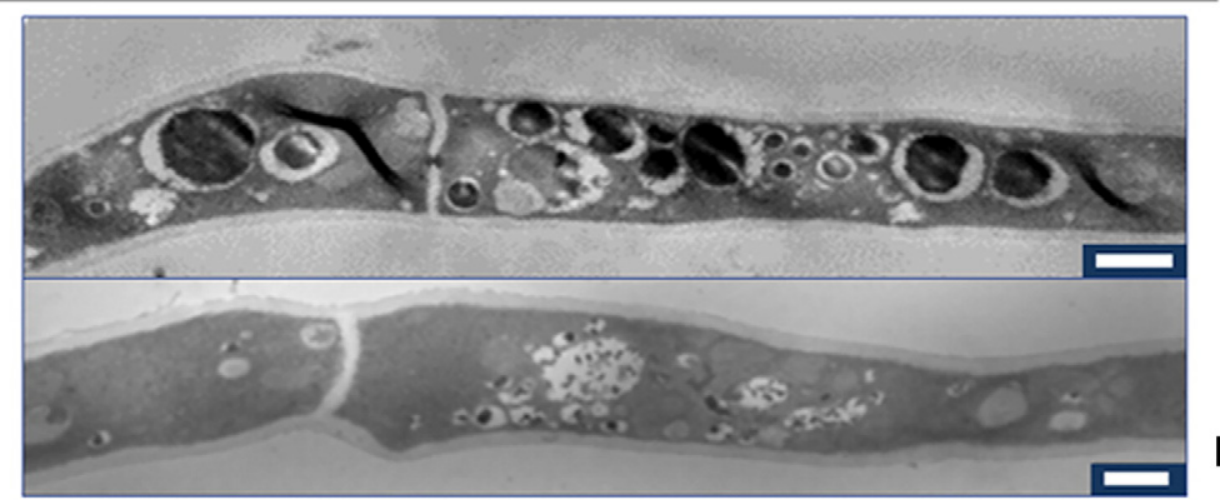

Figure 1. NO-np inhibit Trichophyton rubrum in vitro. (A) Minimum inhibitory and fungicidal concentrations (MICs and MFCs) of NO-np against T. rubrum reference strains (ATCC MYA-4438; ATCC 28189) and a clinical strain performed in triplicate. C-np denotes control (empty) nanoparticles. Quality control tests were performed with terbinafine (TRB) and fluconazole (FLZ), as proposed by the Clinical and Laboratory Standards Institute. ${ }^{12}$ (B) TEM of untreated $T$. rubrum reference strain (upper panel) versus the same strain with $10 \mathrm{mg} / \mathrm{mL}$ NO-np (lower panel) shows accumulation of material suggestive of intracellular vesicles and black granules within the vacuoles at the MIC of NO-np. Scale bars $=1 \mu \mathrm{m}$. 

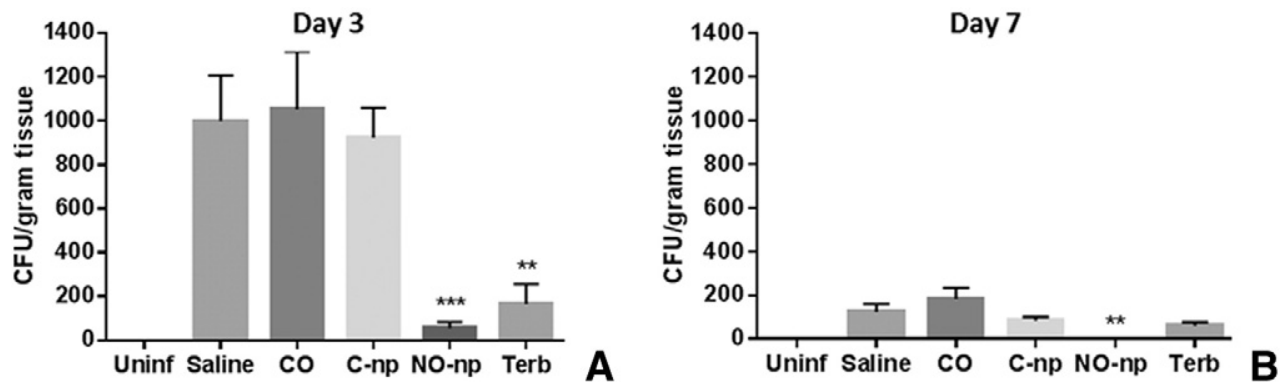

Figure 2. NO-np decrease fungal burden in a murine model of dermal dermatophytosis. Quantification of colony forming units (CFUs) from mouse tissue following 3 (A) and 7 (B) days of treatment. Groups include uninfected mice (Uninf), saline, coconut oil (CO), control (empty) nanoparticles (C-np), NO-np and terbinafine $1 \%$ (Terb) treated mice. $* P<0.05, * * P<0.01,{ }^{* * *} P<0.001 . \mathrm{N}=14$. Error bars denote SEM.

from homogenized tissue using a BD Cytometric Bead Array Mouse Cytokine Kit and BD LSR II flow cytometer (BD Biosciences, San Jose, CA). Data were analyzed with FlowJo software (FlowJo, Ashland, OR). All procedures for animal experimentation were approved by the Institutional Animal Care and Use Committee at Albert Einstein College of Medicine (protocol \#20150403), and international standards were followed to ensure humane treatment of animals.

\section{Results}

The MICs of NO-np for the T. rubrum reference strains and clinical isolate were 10 and $5 \mathrm{mg} / \mathrm{mL}$, respectively. MFC results demonstrated fungistatic behavior of NO-np, and empty nanoparticles did not demonstrate antifungal activity at concentrations up to $20 \mathrm{mg} / \mathrm{mL}$ (Figure 1, A). On TEM, accumulation of material suggestive of intracellular vesicles and black granules within vacuoles was observed in the fungal cells following incubation with the MIC of NO-np (Figure 1, B).

CFU quantification revealed decreased fungal burden in NO-np treated mice compared to saline controls following 3 and 7 days of treatment (Figure 2, $A$ and $B$ ). After 7 days, no fungal growth was observed only in the NO-np group. Significant decreases in IL-2, 6, 10 and TNF $\alpha$ (Figure 3-D) were detected in NO-np treated mice, whereas terbinafine led to decreased IL- 6 only.

\section{Discussion}

Our results present topical NO-np as a potential treatment for dermal T. rubrum infection. NO exerts direct fungistatic activity via DNA damage, lipid peroxidation, and enzyme inactivation, ${ }^{3}$ as well as indirect activity via upregulation of macrophage phagocytic activity. ${ }^{17}$ Of note, NO-np exhibit trans-nitrosylation
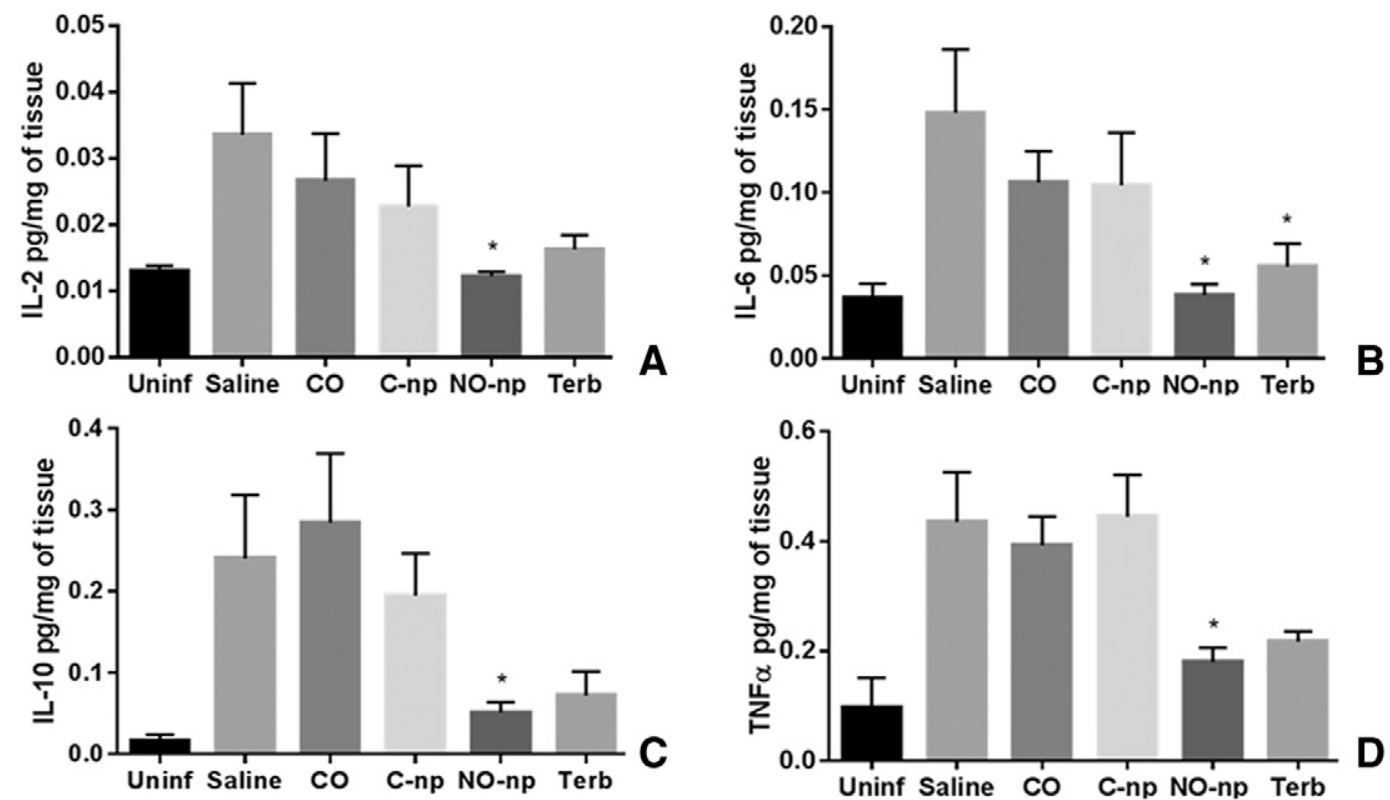

Figure 3. NO-np attenuates the host inflammatory response in a murine model of dermal dermatophytosis. IL-2 (A), 6 (B), 10 (C) and TNF $\alpha$ (D) as measured by enzyme linked immunosorbent assay following 3 days of treatment. Groups include uninfected mice (Uninf), saline, coconut oil (CO), control (empty) nanoparticles (C-np), NO-np and terbinafine $1 \%$ (Terb) treated mice. ${ }^{*} P<0.05, * * P<0.01,{ }^{*} * *<0.001 . \mathrm{N}=7$. Error bars denote SEM. 
activity in addition to NO release, which likely explains the significant effects visualized on TEM. ${ }^{18}$

In addition to fungal death, NO-np were associated with decreased IL-2, 6, 10 and TNF $\alpha$, consistent with a prior study in which exogenous NO downregulated TNF $\alpha$ and IL-2 from murine macrophages and Th1 cells, respectively, without affecting Th2 cytokines. ${ }^{19}$ As NO is an immunomodulator, significant cytokine attenuation in the NO-np group compared to terbinafine is not surprising. ${ }^{17,20,21}$ Although antifungal activity associated with $\mathrm{TNF} \alpha$ downreguation may seem counterintuitive, another study showed that while T. rubrum infection upregulates $\mathrm{TNF} \alpha$ and IL-10 from murine macrophages, autocrine activity was not observed in this setting. ${ }^{22}$ Rather, the authors postulated, the induction of TNF $\alpha$ may play immunoinflammatory roles favoring fungal growth, thereby enhancing T. rubrum virulence. ${ }^{22,23}$

While cytokines' roles in T. rubrum infection and NO's complex, often paradoxical immunomodulatory functions continue to be investigated, our study demonstrates $95 \%$ T. rubrum clearance by day 3 of NO-np treatment coupled with significant cytokine downregulation, indicating an overall earlier attenuation of the host inflammatory response not observed with terbinafine. Given the enhanced inflammatory milieu and fungal clearance, NO-np may reduce local tissue morbidity associated with dermal dermatophytosis.

The effectiveness of topical NO-np is important given that systemic therapy is currently the only option against dermal dermatophytosis. Future studies should aim to translate these findings to human disease, as patients could benefit from shorter treatment courses, lack of systemic side effects and decreased risk of antimicrobial resistance. Furthermore, additional animal models may be pursued to determine NO-np utility in other models of dermatophytosis, such as tinea unguinum or tinea capitis, where inadequate penetration of conventional agents poses similar barriers to topical therapy.

\section{Acknowledgments}

We thank Dr. Mahmoud A. Ghannoum of Case Western Reserve University for providing the T. rubrum ATCC MYA-4438 strain.

\section{References}

1. Gupta AK, Cooper EA. Update in antifungal therapy of dermatophytosis. Mycopathologia 2008;166(5-6):353-67.

2. Akhtar N, Verma A, Pathak K. Topical delivery of drugs for the effective treatment of fungal infections of skin. Curr Pharm Des 2015;21(20):2892-913.

3. De Groote MA, Fang FC. NO inhibitions: antimicrobial properties of nitric oxide. Clin Infect Dis 1995;21(Supplement 2):S162-5.

4. Qin M, Landriscina A, Rosen JM, Wei G, Kao S, Olcott W, et al. Nitric oxide-releasing nanoparticles prevent Propionibacterium acnesinduced inflammation by both clearing the organism and inhibiting microbial stimulation of the innate immune response. J Invest Dermatol 2015;135(11):2723-31.

5. Han G, Friedman AJ, Friedman JM. Nitric oxide releasing nanoparticle synthesis and characterization. Methods Mol Biol 2011:187-95.
6. Friedman AJ, Han G, Navati MS, Chacko M, Gunther L, Alfieri A, et al. Sustained release nitric oxide releasing nanoparticles: characterization of a novel delivery platform based on nitrite containing hydrogel/glass composites. Nitric Oxide 2008;19(1):12-20.

7. Tar M, Cabrales P, Navati M, Adler B, Nacharaju P, Friedman AJ, et al. Topically applied NO-releasing nanoparticles can increase intracorporal pressure and elicit spontaneous erections in a rat model of radical prostatectomy. J Sex Med 2014;11(12):2903-14.

8. Landriscina A, Rosen J, Blecher-Paz K, Long L, Ghannoum MA, Nosanchuk JD, et al. Nitric oxide-releasing nanoparticles as a treatment for cutaneous dermatophyte infections. Sci Lett 2015;4:193.

9. Macherla C, Sanchez DA, Ahmadi MS, Vellozzi EM, Friedman AJ, Nosanchuk JD, et al. Nitric oxide releasing nanoparticles for treatment of Candida albicans burn infections. Front Microbiol 2012;3:193.

10. Friedman A, Blecher K, Sanchez D, Tuckman-Vernon C, Gialanella P, Friedman JM, et al. Susceptibility of gram-positive and-negative bacteria to novel nitric oxide-releasing nanoparticle technology. Virulence 2011;2(3):217-21.

11. Cabrales P, Han G, Roche C, Nacharaju P, Friedman AJ, Friedman JM. Sustained release nitric oxide from long-lived circulating nanoparticles. Free Radic Biol Med 2010;49(4):530-8.

12. Han G, Martinez LR, Mihu MR, Friedman AJ, Friedman JM, Nosanchuk JD. Nitric oxide releasing nanoparticles are therapeutic for Staphylococcus aureus abscesses in a murine model of infection. PLoS One 2009;4(11):e7804.

13. Schairer D, Martinez LR, Blecher K, Chouake J, Nacharaju P, Gialanella $\mathrm{P}$, et al. Nitric oxide nanoparticles: pre-clinical utility as a therapeutic for intramuscular abscesses. Virulence 2012;3(1):62-7.

14. CLSI. Reference Method for Broth Dilution Antifungal Susceptibility Testing of Filamentous Fungi; Approved Standard. 2nd ed. Wayne, PA: Clinical and Laboratory Standards Institute (CLSI); 2008.

15. Ghannoum M, Isham N, Long L. In vitro antifungal activity of ME1111, a new topical agent for onychomycosis, against clinical isolates of dermatophytes. Antimicrob Agents Chemother 2015;59(9):5154-8.

16. Baltazar LM, Santos PC, Paula TP, Rachid MA, Cisalpino PS, Souza DG, et al. IFN- $\gamma$ impairs Trichophyton rubrum proliferation in a murine model of dermatophytosis through the production of IL-1 $\beta$ and reactive oxygen species. Med Mycol 2014 [myt011].

17. Tümer C, Bilgin HM, Obay BD, Diken H, Atmaca M, Kelle M. Effect of nitric oxide on phagocytic activity of lipopolysaccharide-induced macrophages: possible role of exogenous L-arginine. Cell Biol Int 2007;31(6):565-9.

18. Friedman AJ, Blecher K, Schairer D, Tuckman-Vernon C, Nacharaju P, Sanchez D, et al. Improved antimicrobial efficacy with nitric oxide releasing nanoparticle generated S-nitrosoglutathione. Nitric Oxide 2011;25(4):381-6.

19. Eun JS, Suh YH, Kim DK, Jeon H. Regulation of cytokine production by exogenous nitric oxide in murine splenocyte and peritoneal macrophage. Arch Pharm Res 2000;23(5):531-4.

20. Majumdar S, Gupta R, Dogra N. Interferon- $\boldsymbol{\gamma}$ - and lipopolysaccharideinduced tumor necrosis factor- $\alpha$ is required for nitric oxide production: tumor necrosis factor- $\alpha$ and nitric oxide are independently involved in the killing of Mycobacterium microti in interferon- $\gamma$-and lipopolysaccharidetreated J774A. 1 cells. Folia Microbiol 2000;45(5):457-63.

21. Eigler A, Moeller J, Endres S. Exogenous and endogenous nitric oxide attenuates tumor necrosis factor synthesis in the murine macrophage cell line RAW 264.7. J Immunol 1995;154(8):4048-54.

22. Campos M, Russo M, Gomes E, Almeida S. Stimulation, inhibition and death of macrophages infected with Trichophyton rubrum. Microbes Infect 2006;8(2):372-9.

23. Engele M, Stößel E, Castiglione K, et al. Induction of TNF in human alveolar macrophages as a potential evasion mechanism of virulent mycobacterium tuberculosis. J Immunol 2002;168(3):1328-37. 